\title{
Interface Problem in Holonomic Elastoplasticity
}

\author{
Carsten Carstensen \\ Institut für Angewandte Mathematik, Universität Hannover, Welfengarten 1, D-3000 Hannover, \\ FRG
}

Communicated by E. Meister

\begin{abstract}
The three-dimensional interface problem with the homogeneous Lamé system in an unbounded exterior domain and holonomic material behaviour in a bounded interior Lipschitz domain is considered. Existence and uniqueness of solutions of the interface problem are obtained rewriting the exterior problem in terms of boundary integral operators following the symmetric coupling procedure.

The numerical approximation of the solutions consists in coupling of the boundary element method (BEM) and the finite element method (FEM). A Céa-like error estimate is presented for the discrete solutions of the numerical procedure proving its convergence.
\end{abstract}

\section{Introduction}

This paper is concerned with interface (or transmission) problems in solid mechanics which consist of a non-linear problem of holonomic elastoplasticity [20-22] in a bounded Lipschitz domain $\Omega$ and the homogeneous linear elasticity problem in an unbounded exterior domain $\Omega_{2}$. The numerical treatment of such interface problems combines BEM - living on the interface $\Gamma=\bar{\Omega} \cap \bar{\Omega}_{2}$ - and FEM - with a triangulation of $\Omega$.

The coupling of BEM and FEM was introduced by engineers and mathematically justified later. The first papers by Brezzi and Johnson [2] and Johnson and Nedelec [18] present a mathematical explanation for the so-called direct boundary integral method, further extended by Wendland, cf. [23]. Bielak and MacCamy [1] study the so-called single-layer ansatz. These coupling procedures are successful as far as the double-layer potential is compact. In the case of elasticity or if the interface $\Gamma$ is not smooth, the convergence proof generally fails; see however [23] for some special results.

Costabel [4] and Han [15] propose modifications of the coupling of FEM and BEM taking the tractions into account. Further progress is obtained by Costabel and Stephan [10] and Gatica and Hsiao [12,13], treating the coupling procedure also for non-linear problems in $\Omega$ of monotone type. Gatica and Hsiao consider Han's method and can directly apply the theory of monotone operators, while Costabel and Stephan extend the so-called Costabel's symmetric coupling of FEM and BEM which leads to a saddle point problem. 
The interface problem (IP) is formulated in section 2. Following the symmetric coupling procedure an equivalent formulation $(\mathrm{P})$ with boundary integral operators is derived in section 3 . In section 4 we derive some useful equivalent formulations of the problem (P). Existence and uniqueness of solutions of the problem (P), and hence of the interface problem (IP), is proved in section 5. Finally, the numerical approximation with finite and boundary elements is treated in section 6 , where a Céa-like error estimate is proved.

\section{Interface problem}

Let $\Omega_{0} \subset \Omega_{1} \subset \mathbb{R}^{3}$ be bounded Lipschitz domains in three dimensions such that $\Omega_{0}$ lies compactly in $\Omega_{1}$. Then $\Omega:=\Omega_{1} \backslash \bar{\Omega}_{0}$ is the interior domain and $\Omega_{2}:=\mathbb{R}^{3} \backslash \bar{\Omega}_{1}$ is the exterior domain.

The boundary of $\Omega$ is divided into two parts, namely the interior boundary $\Gamma_{0}:=\partial \Omega_{0}$ and the interface $\Gamma:=\partial \Omega_{1}$, cf. Fig. 1. We consider Dirichlet, Neumann or mixed boundary conditions on $\Gamma_{0}$ and allow the case $\Gamma_{0}=\emptyset$ (whence $\Omega_{0}=\emptyset$ ). Let $\bar{t} \in L^{2}\left(\Gamma_{p} ; \mathbb{R}^{3}\right)$ be a given applied surface force, where $\Gamma_{0}=\bar{\Gamma}_{p} \cup \bar{\Gamma}_{u}$ with $\Gamma_{p} \cap \Gamma_{u}=\emptyset$.

The exterior problem is the homogeneous Lamé system of linear elasticity $[9,10]$

$$
\Delta^{*} u:=-\mu_{2} \Delta u-\left(\lambda_{2}+\mu_{2}\right) \operatorname{grad} \operatorname{div} u=0 \text { in } \Omega_{2},
$$

with $\Delta=$ divgrad denoting the Laplace operator, $\mu_{2}, \lambda_{2}$ being the positive Lamé constants.

Given a smooth vector field $u_{2}$, its Cauchy data on the boundary $\Gamma$ are

$$
\left(\left.u_{2}\right|_{\Gamma},\left.T_{2}\left(u_{2}\right)\right|_{\Gamma}\right)
$$

where $T_{2}\left(u_{2}\right)$ is the conormal derivative defined by

$$
T_{2}\left(u_{2}\right):=2 \mu_{2} \partial_{n} u_{2}+\lambda_{2} n \operatorname{div} u_{2}+\mu_{2} n \times \operatorname{curl} u_{2} .
$$

$\partial_{n}$ denotes the normal derivative, $n$ being the unit normal pointing into $\Omega_{2}$, cf. Fig. 1 .

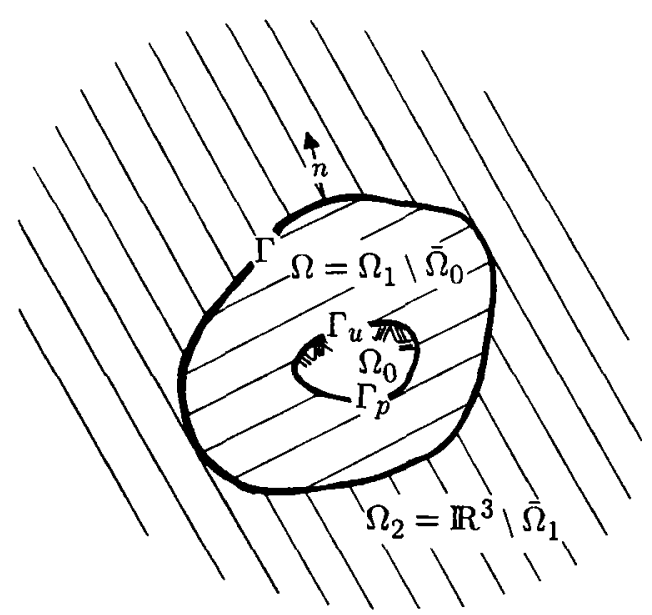

Fig. 1 
Due to the trace lemma $\left.u_{2}\right|_{\Gamma} \in H^{1 / 2}$ whenever $u_{2} \in H_{\text {loc }}^{1}\left(\Omega_{2} ; \mathbb{R}^{3}\right), H_{\text {loc }}^{1}\left(\Omega_{2} ; \mathbb{R}^{3}\right)$ denoting the displacements of locally finite energy.

As e.g. in [8-10] the traction $\left.T_{2}\left(u_{2}\right)\right|_{\Gamma}$ can be defined via the first Green formula. In order to do this, we introduce the following notation:

$$
a_{i j k l}:=\lambda_{2} \delta_{i j} \delta_{k l}+\mu_{2}\left(\delta_{i k} \delta_{j l}+\delta_{i l} \delta_{j k}\right),
$$

where $\delta_{i j}=1$ for $i=j$ and $\delta_{i j}=0$ for $i \neq j$. The strain tensor $\varepsilon(u)$ is defined by

$$
\varepsilon_{i j}(u):=\frac{1}{2}\left(u_{i, j}+u_{j, i}\right)
$$

where $\left(u_{i, j}\right):=\left(u_{i, j}\right)_{i, j=1,2,3}:=\operatorname{grad} u$. The brackets $\langle\cdot, \cdot\rangle$ always denote the duality between $H^{1 / 2}:=H^{1 / 2}\left(\Gamma ; \mathbb{R}^{3}\right)$ and $H^{-1 / 2}:=H^{-1 / 2}\left(\Gamma ; \mathbb{R}^{3}\right)=\left(H^{1 / 2}\right)^{*}$; such that for $v \in H^{1 / 2}$ and $w \in L^{2}\left(\Gamma ; \mathbb{R}^{3}\right)$

$$
\langle w, v\rangle=\int_{\Gamma} u \cdot v \mathrm{~d} \Gamma .
$$

Lemma 1 ([10]). Let $u_{2} \in H_{\text {loc }}^{1}\left(\Omega_{2}, \mathbb{R}^{3}\right)$ with $\Delta^{*} u_{2} \in L_{\text {loc }}^{2}\left(\Omega_{2}, \mathbb{R}^{3}\right)$. Then $\left.T_{2}\left(u_{2}\right)\right|_{\Gamma}$ $\in H^{-1 / 2}\left(\Gamma, \mathbb{R}^{3}\right)$ is defined by

$$
\int_{\Omega_{2}} \Delta^{*} u_{2} v \mathrm{~d} \Omega_{2}=\left\langle T_{2} u_{2},\left.v\right|_{\Gamma}\right\rangle+\Phi_{2}\left(u_{2}, v\right)
$$

for any $v \in H^{1}\left(\Omega_{2} ; \mathbb{R}^{3}\right)$ with compact support and

$$
\Phi_{2}\left(u_{2}, v\right)=\int_{\Omega_{2}} \sum_{i j k l=1}^{3} a_{i j k l} \varepsilon_{k l}\left(u_{2}\right) \varepsilon_{i j}(v) \mathrm{d} \Omega_{2} .
$$

According to Lemma 1 the Cauchy data of a function $u_{2} \in H_{\mathrm{loc}}^{1}\left(\Omega_{2}, \mathbb{R}^{n}\right)$ with $\Delta^{*} u_{2}=0$ satisfy

$$
\left(\left.u_{2}\right|_{\Gamma},\left.T_{2}\left(u_{2}\right)\right|_{\Gamma}\right) \in H^{1 / 2} \times H^{-1 / 2}:=H^{1 / 2}\left(\Gamma ; \mathbb{R}^{3}\right) \times H^{-1 / 2}\left(\Gamma ; \mathbb{R}^{3}\right) .
$$

Note that in (3), $v$ must have a compact support. In order to allow $v \in H^{1}\left(\Omega_{2} ; \mathbb{R}^{3}\right)$, a boundary condition at infinity is required. Following e.g. $[8-10,12,17,19]$, we consider solutions which are regular at infinity, which means in the three-dimensional case that $u_{2}$ satisfies the Sommerfeld's radiation condition

$$
u_{2}=o\left(\frac{1}{|x|}\right) \text { as }|x| \rightarrow \infty .
$$

Then, the exterior problem consists in finding $u_{2} \in \mathscr{L}_{2}$,

$$
\mathscr{L}_{2}:=\left\{u_{2} \in H_{\text {loc }}^{1}\left(\Omega_{2} ; \mathbb{R}^{3}\right): u_{2} \text { satisfies (4) and } \Delta^{*} u_{2}=0\right\},
$$

subject to some interface conditions concerning the Cauchy data $\left(\left.u_{2}\right|_{\Gamma}, T_{2} u_{2}\right)$ of $u_{2}$.

The non-linear interior problem of holonomic elastoplasticity is considered in [20-22] and leads to a variational inequality.

The strong form of local equilibrium is

$$
\begin{aligned}
\sigma=\sigma^{\mathrm{T}} & \text { in } \Omega, \\
\operatorname{div} \sigma+f=0 & \text { in } \Omega, \\
\sigma n=t & \text { on } \Gamma, \\
\sigma n=\bar{t} & \text { on } \Gamma_{p},
\end{aligned}
$$


where $\sigma$ is the symmetric stress field, $f$ is the vector field of given body forces, $\bar{t}$ is a given surface traction, $t$ are the (unknown) tractions on $\Gamma$ and $n$ is the outer normal vector along the interface as well as $\Gamma_{p}$.

In order to state the weak form of the equilibrium conditions, let

$$
H:=H_{u}^{1}\left(\Omega ; \mathbb{R}^{3}\right):=\left\{u \in H^{1}\left(\Omega ; \mathbb{R}^{3}\right):\left.u\right|_{\Gamma_{u}}=0\right\},
$$

$H^{*}$ being the dual of $H$, and let

$$
L:=L^{2}\left(\Omega ; \mathbb{R}_{\mathrm{sym}}^{3 \times 3}\right)
$$

denote the Lebesgue space with values in the six-dimensional real vector space $\mathbb{R}_{\text {sym }}^{3 \times 3}$ of symmetric three-dimensional matrices. $L$ is identified with its dual, i.e. $L^{*}=L$.

For real Banach spaces $X$ and $Y$, let $\mathscr{L}(X ; Y)$ denote the real vector space of bounded linear mappings from $X$ into $Y$. If $x \in X$ and $Y \subseteq X$ then $\operatorname{dist}_{X}(x, Y):=\inf \left\{\|x-y\|_{X}: y \in Y\right\}$ denotes the distance of $x$ to $Y$.

Then, the trace mapping

$$
\gamma:=\left.\cdot\right|_{\Gamma} \in \mathscr{L}\left(H ; H^{1 / 2}\right), \quad \gamma u:=\left.u\right|_{\Gamma} \quad(u \in H),
$$

has the dual $\gamma^{*} \in \mathscr{L}\left(H^{-1 / 2} ; H^{*}\right)$. Let the mapping

$$
\varepsilon \in \mathscr{L}(H ; L)
$$

be defined by (2), $\varepsilon^{*} \in \mathscr{L}\left(L, H^{*}\right)$ being the dual of $\varepsilon$.

The weak form of the equilibrium condition is obtained as usual: Take a test function $v \in H:=H_{u}^{1}\left(\Omega, \mathbb{R}^{3}\right)$, multiply div $\sigma+f=0$ with $v$, integrate over $\Omega$ to obtain after integration by parts (Green's formulas) using e.g. $t=\sigma n$ on $\partial \Omega=\Gamma \cup \Gamma_{0}$, etc.

$$
\int_{\Gamma} t^{\mathrm{T}} \cdot v \mathrm{~d} \Gamma+\int_{\Gamma_{p}} \bar{t}^{\mathrm{T}} \cdot v \mathrm{~d} \Gamma-\int_{\Omega} \sigma: \operatorname{grad} v \mathrm{~d} \Omega+\int_{\Omega} v^{\mathrm{T}} \cdot f \mathrm{~d} \Omega=0 .
$$

Since $\sigma: \operatorname{grad} v=\sigma: \varepsilon v$, ':' being the scalar product in $\mathbb{R}^{3 \times 3}$, the last formula gives the weak form of equilibrium

$$
\varepsilon^{*} \sigma=\gamma^{*} t+b
$$

for $\sigma \in L, t \in H^{-1 / 2}, b \in H^{*}$.

Besides the equilibrium, constitutive relations are required. The stress-strain relation is given by

$$
\sigma=A(\varepsilon u-p)
$$

where $p \in L$ is a new internal variable which may be regarded as the plastic part $p$ of the total strain $\varepsilon u$. $A \in \mathscr{L}(L ; L)$ describes linear elasticity, is symmetric and positivedefinite, i.e. there exists a constant $c>0$ with

$$
\langle A \xi, \xi\rangle_{H} \geqslant c \cdot\|\xi\|_{L}^{2} \text {. }
$$

Since an additional internal variable $p$ is under consideration a further constitutive relation for $p$ is required. Following $[16,20,22]$, we consider

$$
\sigma-\eta p \in \partial \tilde{W}\left(p-p_{0}\right) \text { in } \Omega
$$

where $p_{0} \in L$ is prescribed and $\tilde{W}(q):=k \cdot|q|$ for any $q \in \mathbb{R}_{\text {sym }}^{3 \times 3}$, $|q|=\sqrt{q: q}=\sqrt{\sum_{i, j=1,2,3} q_{i j}^{2}}$. Assume that $k, \eta \in L^{\infty}(\Omega, \mathbb{R})$ and that there exist 
constants $\eta_{1}, \eta_{2}, k_{1}, k_{2}$ with

$$
0<\eta_{1} \leqslant \eta(x) \leqslant \eta_{2}<\infty \text { and } 0<k_{1} \leqslant k(x) \leqslant k_{2}<\infty
$$

for a.e. $x \in \Omega$. $\eta$ is the hardening parameter while $k$ and $p_{0}$ describe the yield function. $\tilde{W}: \mathbb{R}_{\text {sym }}^{3 \times 3} \rightarrow \mathbb{R}$ represents the non-differentiable part of the minimum plastic work function. $\partial \tilde{W}$ denotes the subdifferential defined through

$$
\partial \tilde{W}(r)=\left\{\sigma \in \mathbb{R}_{\mathrm{sym}}^{3 \times 3}: \forall q \in \mathbb{R}_{\mathrm{sym}}^{3 \times 3}, \tilde{W}(q)-\tilde{W}(r) \geqslant \sigma:(q-r)\right\},
$$

$r \in \mathbb{R}_{\text {sym }}^{3 \times 3}$. Define

$$
j: L \rightarrow \mathbb{R}, \quad q \mapsto \int_{\Omega} \tilde{W}(q) \mathrm{d} \Omega=\int_{\Omega} k \cdot|q| \mathrm{d} \Omega .
$$

Then, $j$ is convex and Lipschitz-continuous (cf. [20, Lemma 2.3]). Let $\partial j$ be the subdifferential of $j$ in $L$, i.e.

$$
\partial j(p)=\left\{q \in L: \forall r \in L, j(p)-j(r) \leqslant\langle q, p-r\rangle_{L}\right\} ;
$$

note that we identify $L$ with its dual $L^{*}$. Then the weak form of $(10)$ reads

$$
\sigma-\eta p \in \partial j\left(p-p_{0}\right) \text {. }
$$

Definition 1. Given $b \in H^{*}$ and $p_{0} \in L$, the interface problem (IP) consists in finding functions $u \in H, u_{2} \in \mathscr{L}_{2}, \sigma \in L, p \in L$ satisfying (8), (9), (13) and $t \in H^{-1 / 2}$ with

$$
\gamma u=\left.u_{2}\right|_{\Gamma}, \quad t=\left.T_{2}\left(u_{2}\right)\right|_{\Gamma}
$$

Remark 1. We neglected initial values $\sigma_{0}$ and $u_{0}$ from $[16,20,22]$ for convenience of notation. It can be observed in e.g. [20, Theorem 2.1] that $\sigma_{0} \neq 0, u_{0} \neq 0$ only concerns the constant or linear terms, while $p_{0} \neq 0$ is used in the non-linear part $\partial \tilde{W}\left(p-p_{0}\right)$. Hence, $\sigma_{0}=0, u_{0}=0$ is no essential restriction.

\section{Formulation with houndary integral operators}

The interface problem can be rewritten in terms of boundary integral operators leading to non-local boundary conditions. For the Lamé operator the fundamental solution $G_{2}$ with the kernel $G_{2}(x, y)$, called Kelvin-matrix, is well known, i.e.

$$
G_{2}(x, y)=\frac{\lambda_{2}+3 \mu_{2}}{8 \pi \mu_{2}\left(\lambda_{2}+2 \mu_{2}\right)}\left\{\frac{1}{|x-y|} I+\frac{\lambda_{2}+\mu_{2}}{\lambda_{2}+3 \mu_{2}} \frac{(x-y)(x-y)^{\mathrm{T}}}{|x-y|^{3}}\right\}
$$

for the three-dimensional case. $I$ is the unit matrix and $T$ denotes the transposed matrix. Since $G_{2}$ is analytic in $\mathbb{R}^{3} \times \mathbb{R}^{3}$ without the diagonal, we may define its traction

$$
T_{2}(x, y):=T_{2, y}\left(G_{2}(x, y)\right)^{\mathrm{T}}, \quad x \neq y .
$$

Due to the second Green formula (see Lemma 1), the following Somigliana representation formula for $x \in \mathbf{\Omega}_{2}$,

$$
u_{2}(x)=\left\langle T_{2}(x, \cdot), v\right\rangle-\left\langle G_{2}(x, \cdot), \phi\right\rangle,
$$

is proved for Lipschitz domains in [5]. Equation (15) holds for all $u_{2} \in H_{\text {loc }}^{1}\left(\Omega_{2}\right)$ with compact support satisfying (1) and $v=\left.u_{2}\right|_{\Gamma}, \phi=\left.T_{2}\left(u_{2}\right)\right|_{\Gamma}$. 
For any $x \in \Omega_{2},(15)$ can be differentiated giving a representation formula for the stresses $T_{2}\left(u_{2}\right)$. By using the classical jump relations for $x \rightarrow \Gamma$ and inserting the Cauchy data into these formulas, one obtains on $\Gamma$

$$
\left(\begin{array}{l}
v \\
\phi
\end{array}\right)=\mathscr{C}_{2} \cdot\left(\begin{array}{l}
v \\
\phi
\end{array}\right),
$$

where the Calderón projector

$$
\mathscr{C}_{2}=\left(\begin{array}{cc}
\frac{1}{2}+\Lambda_{2} & -V_{2} \\
-D_{2} & \frac{1}{2}-\Lambda_{2}^{\prime}
\end{array}\right)
$$

is defined through

$$
\begin{aligned}
\left(V_{2} \phi\right)(x) & =\left\langle G_{2}(x, \cdot), \phi\right\rangle, \\
\left(\Lambda_{2} v\right)(x) & =\left\langle T_{2}(x, \cdot), v\right\rangle, \\
\left(D_{2} v\right)(x) & =-T_{2 \cdot x}\left(\left\langle T_{2}(x, \cdot), v\right\rangle\right), \\
\left(\Lambda_{2}^{\prime} \phi\right)(x) & =-T_{2 \cdot x}\left(\left\langle G_{2}(x, \cdot), \phi\right\rangle\right) \quad(x \in \Gamma) .
\end{aligned}
$$

$V_{2}$ is the single-layer potential, $\Lambda_{2}$ is the double-layer potential with its dual $\Lambda_{2}^{\prime}$ and $D_{2}$ is the hypersingular operator.

The next lemma recalls some properties of the above operators as their domain, continuity and ellipticity from [4, Lemma 4.1], [5, Theorem 1] and [10, Lemma 4.5]; see also [7] and [8, Lemma 3.9] for related results.

Lemma $2([5,10])$. Set $H^{1 / 2}:=H^{1 / 2}\left(\Gamma, \mathbb{R}^{3}\right), H^{-1 / 2}:=H^{-1 / 2}\left(\Gamma, \mathbb{R}^{3}\right)$. Then

$$
\begin{aligned}
& V_{2} \in \mathscr{L}\left(H^{-1 / 2} ; H^{1 / 2}\right), \\
& \Lambda_{2} \in \mathscr{L}\left(H^{1 / 2} ; H^{1 / 2}\right) \\
& \Lambda_{2}^{\prime} \in \mathscr{L}\left(H^{-1 / 2} ; H^{-1 / 2}\right), \\
& D_{2} \in \mathscr{L}\left(H^{1 / 2} ; H^{-1 / 2}\right) .
\end{aligned}
$$

$D_{2}$ is positive-semi-definite and $V_{2}$ is positive-definite, i.e. there exists a constant $c>0$ such that for all $v \in H^{1 / 2}$ and all $\phi \in H^{-1 / 2}$ there holds

$$
\left\langle D_{2} v, v\right\rangle \geqslant 0 \text { and }\left\langle\phi, V_{2} \phi\right\rangle \geqslant c\|\phi\|_{H^{-1 / 2}}^{2} \text {. }
$$

$D_{2}$ and $V_{2}$ are symmetric, $\Lambda^{\prime}$ is the dual of $\Lambda$.

The relations between the Calderón projector $\mathscr{C}_{2} \in \mathscr{L}\left(H^{1 / 2} \times H^{-1 / 2} ; H^{1 / 2} \times H^{-1 / 2}\right)$ and the Cauchy data of a function in $\mathscr{L}_{2}$ are recalled in the next lemma.

Lemma $3([9,10])$. (i) If $u_{2} \in \mathscr{L}_{2}$ then (15) holds for $v:=\left.u_{2}\right|_{\Gamma} \in H^{1 / 2}:=H^{1 / 2}\left(\Gamma, \mathbb{R}^{3}\right)$ and $\phi:=\left.T_{2}\left(u_{2}\right)\right|_{\Gamma} \in H^{-1 / 2}:=H^{-1 / 2}\left(\Gamma, \mathbb{R}^{3}\right)$.

(ii) For any $v \in H^{1 / 2}$ and $\phi \in H^{-1 / 2}$ the vector field $u_{2}$ defined via (15) belongs to $\mathscr{L}_{2}$.

(iii) For $(v, \phi) \in H^{1 / 2} \times H^{-1 / 2}$ the following statements (a) and (b) are equivalent.

(a) $(v, \phi)$ are Cauchy data of some $u_{2} \in \mathscr{L}_{2}$, i.e. $v=\left.u_{2}\right|_{\Gamma}, \phi=\left.T_{2}\left(u_{2}\right)\right|_{\Gamma}$ for some $u_{2} \in \mathscr{L}_{2}$.

(b) $(v, \phi)$ satisfies $(16)$.

(iv) The Calderon projector is a projection in $H^{1 / 2} \times H^{-1 / 2}$ onto its subspace of Cauchy data of weak solutions in $\mathscr{L}_{2}$, i.e. onto $\left\{\left(\left.u_{2}\right|_{\Gamma},\left.T_{2}\left(u_{2}\right)\right|_{\Gamma}\right): u_{2} \in \mathscr{L}_{2}\right\}$. 
We are now in a position to prove the following equivalence result concerning problem (P).

Definition 2. Given $b \in H^{*}$ and $p_{0} \in L$ the problem $(P)$ consists in finding functions $u \in H$ and $p \in L$ satisfying

$$
\begin{aligned}
& \left(\varepsilon^{*} A \varepsilon+S\right) u=b+\varepsilon^{*} A p, \\
& A \varepsilon u-(A+\eta I) p \in \partial j\left(p-p_{0}\right),
\end{aligned}
$$

where $I$ denotes identity in $L$ and

$$
\begin{aligned}
S_{2} & :=D_{2}+\left(1 / 2-\Lambda_{2}^{\prime}\right) V_{2}^{-1}\left(1 / 2-\Lambda_{2}\right) \in \mathscr{L}\left(H^{1 / 2} ; H^{-1 / 2}\right), \\
S & :=\gamma^{*} S_{2} \gamma \in \mathscr{L}\left(H ; H^{*}\right) .
\end{aligned}
$$

Theorem 1. $\left(u, u_{2}, \sigma, p\right) \in H \times \mathscr{L}_{2} \times L \times L$ solves the interface problem (IP) if and only if $(u, \sigma, p) \in H \times L \times L$ solves problem $(\mathrm{P})$.

In the latter case, $u_{2}$ is given through (15), where $v=\gamma u, \phi:=V_{2}^{-1}\left(\Lambda_{2}-1 / 2\right) v$.

Proof. Let $\left(u_{1}, \sigma, p, u_{2}\right) \in H \times L \times L \times \mathscr{L}_{2}$ be a solution of the interface problem (IP) of Definition 1. According to Lemma $3(v, t)$ are Cauchy data of some $u_{2} \in \mathscr{L}_{2}$ iff $(v, t)$ satisfies (16) (with $\phi=t$ ), which is equivalent to

$$
V_{2} \phi=\left(\Lambda_{2}-1 / 2\right) v \text { and } \phi=-D_{2} v+\left(1 / 2-\Lambda_{2}^{\prime}\right) \phi .
$$

Since-due to the Lax-Milgram lemma- $V_{2}$ is invertible, (21) is equivalent to

$$
\phi=V_{2}^{-1}\left(\Lambda_{2}-1 / 2\right) v \text { and } \phi=-D_{2} v+\left(1 / 2-\Lambda_{2}^{\prime}\right) V_{2}^{-1}\left(\Lambda_{2}-1 / 2\right) v .
$$

The last equation in (22) is $t=\phi=-S_{2} v$. Substitution of $t=-S_{2} \gamma u$ in (8) and substitution of $\sigma$ from (9) in (8) and (13) prove (17) and (18).

In order to verify the second implication assume that $(u, p) \in H \times L$ solves problem (P). Define $u_{2}$ as in the theorem by (15), where $v=\gamma u, \phi:=V_{2}^{-1}\left(\Lambda_{2}-1 / 2\right) v$. Then, according to Lemma 3(ii), $u_{2} \in \mathscr{L}_{2}$.

Using the jump relations for $x \rightarrow \Gamma$ in (15) (cf. Lemma 3(jv)), we obtain

$$
\left(\begin{array}{c}
\left.u_{2}\right|_{\Gamma} \\
\left.T_{2}\left(u_{2}\right)\right|_{\Gamma}
\end{array}\right)=\mathscr{C}_{2}\left(\begin{array}{c}
v \\
\phi
\end{array}\right)=\left(\begin{array}{c}
1 / 2 v+\Lambda_{2} v-V_{2} \phi \\
-D_{2} v+\left(1 / 2-\Lambda_{2}^{\prime}\right) \phi
\end{array}\right)=\left(\begin{array}{c}
v \\
-S_{2} v
\end{array}\right),
$$

where the last equality follows from the definition of $\phi$ and $S_{2}$. Therefore, one obtains with $t:=-S_{2} v,\left.u_{2}\right|_{\Gamma}=v,\left.T_{2}\left(u_{2}\right)\right|_{\Gamma}=t$.

Define $\sigma$ by (9). The same calculations as in the first part of the proof verify that $\left(u, u_{2}, \sigma, p\right) \in H \times \mathscr{L}_{2} \times L \times L$ solves the interface problem (IP).

Remark 2. $S_{2}$ can be considered as the Schur-complement in

$$
\left(\begin{array}{cc}
D_{2} & \left(1 / 2-\Lambda_{2}^{\prime}\right) \\
\left(1 / 2-\Lambda_{2}\right) & -V_{2}
\end{array}\right)
$$

with respect to the pivot element $-V_{2}$. It is related to one step of Gaussian elimination with the pivot block; as seen in the proof of Theorem $1, S_{2}$ arises eliminating the second variable.

$S_{2}$ is sometimes called Dirichlet-Neumann map or Poincaré-Stecklov operator. 
Remark 3. According to Lemma $2, S_{2} \in \mathscr{L}\left(H^{1 / 2} ; H^{-1 / 2}\right)$, as defined in (19), is symmetric and positive-semi-definite, i.e. for all $v, w \in H^{1 / 2}$ there holds

$$
\left\langle S_{2} v, w\right\rangle=\left\langle S_{2} w, v\right\rangle \text { and }\left\langle S_{2} v, v\right\rangle \geqslant 0 \text {. }
$$

Following the proof of [10, Lemma 4.5], one can see that $S_{2}$ is positive-definite.

Lemma 4 ([6]). $S_{2}$ is positive-definite, i.e. there exists $c>0$ such that for any $v \in H^{1 / 2}$ there holds

$$
\left\langle S_{2} v, v\right\rangle \geqslant c\|v\|_{H^{1 / 2}} .
$$

Proof. As shown in [10, Lemma 4.5], $D_{2}$, and hence $S_{2}$, is a compact perturbation of a positive-definite operator (see also [5]). Thus, since $S_{2}$ is positive-semi-definite, it remains to prove that $v \in H^{1 / 2}$ and $S_{2} v=0$ imply $v=0$.

Given $v \in H^{1 / 2}$ with $0=S_{2} v$, define $u_{2} \in \mathscr{L}_{2}$ by (15) for $\phi=0$. Note that $S_{2} v=0$ implies $D_{2} v=0$ and $\left(\Lambda_{2}-\frac{1}{2}\right) v=0$. Then, as seen in the second part of the proof of Theorem 1, we have $\left.u_{2}\right|_{\Gamma}=v$ and $\left.T_{2}\left(u_{2}\right)\right|_{\Gamma}=\phi=0$. As in the proof of $[9$, Lemma 4.5], (4) allows us to apply the first Green's formula to $u \cdot \Delta^{*} u=0$ in $\Omega_{2}$, which (cf. (3)) leads to

$$
0=\Phi_{2}\left(u_{2}, u_{2}\right)
$$

i.e. $u_{2}$ is a rigid-body motion. Because of (4), this gives $u_{2}=0$.

\section{Equivalent formulations}

Some calculations give further equivalence results (cf. [20, Theorem 2.1] and Theorem 3 below) for which we need that $\varepsilon^{*} \varepsilon+S$ is positive-definite.

Lemma 5. $\varepsilon^{*} \varepsilon+S \in \mathscr{L}\left(H ; H^{*}\right)$ is positive-definite.

Proof. Using Lemma 4 it remains to show that there exists a constant $c_{1}>0$ such that for any $u \in H$

$$
\|\varepsilon u\|_{L}^{2}+\|\gamma u\|_{H^{1 / 2}}^{2} \geqslant c_{1}\|u\|_{H}^{2} .
$$

According to Korn's inequality we have some $c_{2}>0$ such that for any $w \in X_{0}^{\frac{1}{0}}$

$$
\|\varepsilon w\|_{\mathbf{L}}^{2} \geqslant c_{2}\|w\|_{H}^{2} .
$$

Here $X_{0}:=\{u \in H: \varepsilon u=0\}$ consists of rigid-body motions and is a finite-dimensional subspace of $H$ with the orthogonal complement $X_{0}^{\perp}$, see [26, Theorem 62.F] for proofs. We remark that $X_{0}=\{0\}$ if $\Gamma_{u}$ has positive surface measure, in general, we allow $\Gamma_{u}=\emptyset$.

In order to prove (23), assume that it is false. Then, for any positive integer $n$, there exists $u_{n}=v_{n}+w_{n} \in H$ with $\left\|u_{n}\right\|_{H}=1, v_{n} \in X_{0}, w_{n} \in X_{0}^{\perp}$ and

$$
\frac{1}{n} \geqslant\left\|\varepsilon u_{n}\right\|_{L}^{2}+\left\|\gamma u_{n}\right\|_{H^{1 / 2}}^{2} \text {. }
$$

Because of (24) and $\varepsilon u_{n}=\varepsilon w_{n},(25)$ gives

$$
\lim _{n \rightarrow \infty}\left\|w_{n}\right\|_{H}=0
$$


Since $v_{n}$ is a bounded sequence in the finite-dimensional space $X_{0}$, we have a subsequence (again denoted as $\left(v_{n}\right)$ ) which converges, i.e. there exists $v \in X_{0}$, with

$$
\lim _{n \rightarrow \infty} u_{n}=\lim _{n \rightarrow \infty} v_{n}=v, \quad \text { whence }\|v\|_{H}=1 .
$$

By (25), the convergence of (a subsequence of) $\left(u_{n}\right)$ in $H$ implies $\gamma v=0$. Because $v \in X_{0}$ is a rigid-body motion and the interface has positive surface measure, $\left.v\right|_{\Gamma}=0$ implies $v=0$. This contradicts $\|v\|_{H}=1$.

Since $A$ is positive-definite, the lemma implies that $\varepsilon^{*} A \varepsilon+S$ is invertible. Hence, we are now in the position to define

$$
\hat{A}:=A+\eta I-A \varepsilon\left(\varepsilon^{*} A \varepsilon+S\right)^{-1} \varepsilon^{*} A \in \mathscr{L}(L ; L)
$$

and

$$
g:=A \varepsilon\left(\varepsilon^{*} A \varepsilon+S\right)^{-1} b \in L
$$

Using

$$
\mathscr{A}:=\left(\begin{array}{cc}
S+\varepsilon^{*} A \varepsilon & -\varepsilon^{*} A \\
-A \varepsilon & A+\eta I
\end{array}\right) \in \mathscr{L}\left(H \times L ; H^{*} \times L\right),
$$

define also the bilinear form

$$
a:(H \times L) \times(H \times L) \rightarrow \mathbb{R}, \quad\left(\left(\begin{array}{l}
u \\
p
\end{array}\right),\left(\begin{array}{l}
v \\
q
\end{array}\right)\right) \mapsto\left\langle\mathscr{A}\left(\begin{array}{l}
u \\
p
\end{array}\right),\left(\begin{array}{l}
v \\
q
\end{array}\right)\right\rangle_{H \times L}
$$

and the linear form

$$
l: H \times L \rightarrow \mathbb{R},\left(\begin{array}{l}
u \\
p
\end{array}\right) \mapsto\left\langle b+\varepsilon^{*} A p_{0}, u\right\rangle_{H}-\left\langle(A+\eta I) p_{0}, p\right\rangle_{L} .
$$

Note that (17) is equivalent to

$$
u=\left(\varepsilon^{*} A \varepsilon+S\right)^{-1}\left(b+\varepsilon^{*} A p\right) \in H .
$$

Theorem 2. The following statements for $u \in H$ and $p \in L$ are equivalent.

(i) $(u, p) \in H \times L$ solves problem $(\mathrm{P})$.

(ii) $p \in L$ solves

$$
g \in \hat{A} p+\partial j\left(p-p_{0}\right)
$$

and $u$ is given by (31).

(iii) $p \in L$ solves the variational inequality

$$
\left\langle\hat{A} p-g, q-p+p_{0}\right\rangle_{L}+j(q)-j\left(p-p_{0}\right) \geqslant 0 \text { for all } q \in L
$$

and $u$ is given by (31).

(iv) $u \in H$ and $p \in L$ solve the variational inequality

$$
\begin{aligned}
& a\left(\left(\begin{array}{c}
u \\
p-p_{0}
\end{array}\right),\left(\begin{array}{c}
v-u \\
q-p+p_{0}
\end{array}\right)\right)+j(q)-j\left(p-p_{0}\right)-l\left(\left(\begin{array}{c}
v-u \\
q-p+p_{0}
\end{array}\right)\right) \geqslant 0 \\
& \text { for all }\left(\begin{array}{l}
v \\
q
\end{array}\right) \in H \times L .
\end{aligned}
$$


Proof. (i) $\Leftrightarrow$ (ii): Assume that $(u, p)$ solves problem (P). Then, substitution of (31) in (18) yields (32) using the definitions (26) and (27). Conversely, if $p$ solves (32) define $u$ by (31). Then, the same substitutions as in the first part of the proof show that $(u, p)$ solves (P).

(ii) $\Leftrightarrow$ (iii): The equivalence of (33) and (32) follows directly from the definition of the subdifferential.

(iii) $\Leftrightarrow$ (iv): For any $(v, q) \in H \times L$ there holds, using (29) and (30),

$$
\begin{aligned}
& a\left(\left(\begin{array}{c}
u \\
p-p_{0}
\end{array}\right),\left(\begin{array}{c}
v-u \\
q-p+q_{0}
\end{array}\right)\right)+j(q)-j\left(p-p_{0}\right)-l\left(\left(\begin{array}{c}
v-u \\
q-p+p_{0}
\end{array}\right)\right) \\
& =\left\langle\left(S+\varepsilon^{*} A \varepsilon\right) u-\varepsilon^{*} A p-b, v-u\right\rangle_{H} \\
& \quad+\left\langle(A+\eta I) p-A \varepsilon u, q-p+p_{0}\right\rangle_{L}+j(q)-j\left(p-p_{0}\right) .
\end{aligned}
$$

Let $q=p-p_{0}$ and let $v \in H$ be arbitrary and, conversely, let $v=u$ and let $q \in H$ be arbitrary in the last expression to prove that (iv) is equivalent to (31) and

$$
\left\langle(A+\eta I) p-A \varepsilon u, q-p+p_{0}\right\rangle_{L}+j(q)-j\left(p-p_{0}\right) \geqslant 0
$$

for all $q \in L$. Substitution of $u$ from (31) in the last inequality shows that (iv) is equivalent to (31) and (33).

\section{Existence and uniqueness of solutions}

Using convex analysis we prove that the interface problem (IP) has a unique solution. As a crucial point it remains to show that $\hat{A}$ is positive-definite. The proof given here is different from that of [20, Lemma 2.1(a)].

Lemma 6. $\hat{A}$ given in (26) and $\mathscr{A}$ given in (28) are positive-definite.

Proof. Using

$$
\begin{aligned}
& \left\langle\left(\begin{array}{cc}
\varepsilon^{*} A \varepsilon+S & \varepsilon^{*} A \\
A \varepsilon & A
\end{array}\right)\left(\begin{array}{l}
u \\
p
\end{array}\right),\left(\begin{array}{l}
u \\
p
\end{array}\right)\right\rangle_{H \times L} \\
& =\langle S u, u\rangle_{H}+\langle A(\varepsilon u+p),(\varepsilon u+p)\rangle_{L} \geqslant 0
\end{aligned}
$$

and

$$
\begin{aligned}
& \left(\begin{array}{cc}
I & 0 \\
-A \varepsilon\left(\varepsilon^{*} A \varepsilon+S\right)^{-1} & I
\end{array}\right)\left(\begin{array}{cc}
\varepsilon^{*} A \varepsilon+S & \varepsilon^{*} A \\
A \varepsilon & A
\end{array}\right)\left(\begin{array}{cc}
I & -\left(\varepsilon^{*} A \varepsilon+S\right)^{-1} \varepsilon^{*} A \\
0 & I
\end{array}\right) \\
& =\left(\begin{array}{cc}
\varepsilon^{*} A \varepsilon+S & 0 \\
0 & \hat{A}-\eta I
\end{array}\right)
\end{aligned}
$$

( 0 and $I$ denote zero and identity in different spaces), one concludes that $\hat{A}-\eta I$ is positive-semi-definite. Thus, $\hat{A}$ is positive-definite.

The same considerations with

$$
\left(\begin{array}{cc}
\varepsilon^{*} A \varepsilon+S & -\varepsilon^{*} A \\
-A \varepsilon & A
\end{array}\right)
$$

prove that $\mathscr{A}$ is positive-definite. 
Following $[16,20,22]$, existence and uniqueness of the solution of the interface problem (IP) will now be concluded with standard arguments from convex analysis.

Theorem 3. The interface problem (IP) as well as the equivalent problems introduced in Theorems 1 and 2 have unique solutions.

In particular, $p \in L$ solves (32) iff $p$ is the unique minimizer of the functional

$$
\varphi: L \rightarrow \mathbb{R}, \quad q \mapsto\left\langle\frac{1}{2} \hat{A} q-g, q\right\rangle_{L}+j\left(q-p_{0}\right)
$$

$u \in H$ and $p \in L$ solve (34) iff $\left(u, p-p_{0}\right)$ minimizes the functional

$$
\left.J: H \times L \rightarrow \mathbb{R}, \quad(v, q) \mapsto \frac{1}{2} a\left(\left(\begin{array}{l}
v \\
q
\end{array}\right),\left(\begin{array}{l}
v \\
q
\end{array}\right)\right)+j(q)-l\left(\begin{array}{l}
v \\
q
\end{array}\right)\right) .
$$

Proof. Note that $\varphi$ is convex and its subdifferential is equal to

$$
\partial \varphi(p)=\hat{A} p-g+\partial j\left(p-p_{0}\right)
$$

(for a proof cf. e.g. [25, Section $47.5 \mathrm{ff}]$ for the sum rule and the relation to the Gateaux derivative). Since $\varphi$ is strictly convex, continuous and coercive (because $\hat{A}$ is strictly positive), $\varphi$ has a unique minimizer (for a proof cf. e.g. [24, Theorem 25.E]).

It is well known from convex analysis (cf. e.g. [25, Proposition 47.12]) that $p \in L$ minimizes $\varphi$ iff $0 \in \partial \varphi(p)$ which is equivalent to (32). According to Theorems 1 and 2 this implies that the interface problem has a unique solution.

The same arguments used for the calculation of $\partial \varphi$ prove that $0 \in \partial J\left(u, p-p_{0}\right)$ is equivalent to

$$
l-\mathscr{A}\left(\begin{array}{c}
u \\
p-p_{0}
\end{array}\right) \in \partial j\left(p-p_{0}\right)
$$

which is (34).

The following lemma is used in the proof of Theorem 4 and proved in [16, Theorem $2.1]$ for $S=0$. The proof works verbatim for the present case.

Lemma 7. If $(u, p)$ solves problem $(\mathrm{P})$ then there exists a multiplier $\lambda \in L$ with $|\lambda| \leqslant 1$ and $\lambda \cdot\left(p-p_{0}\right)=\left|p-p_{0}\right|$ a.e. in $\Omega$ such that for any $(v, q) \in H \times L$

$$
a\left(\left(\begin{array}{c}
u \\
p-p_{0}
\end{array}\right),\left(\begin{array}{l}
v \\
q
\end{array}\right)\right)+\int_{\Omega} k \lambda q \mathrm{~d} \Omega=l\left(\left(\begin{array}{l}
v \\
q
\end{array}\right)\right) .
$$

\section{Numerical treatment}

The discretization of the problem $(\mathrm{P})$ is described in this section, leading to the coupling of FEM and BEM. Let

$$
\left(H_{h} \times L_{k} \times H_{h}^{-1 / 2}: h \in I\right)
$$

be a family of finite-dimensional subspaces of $H \times L \times H^{-1 / 2}$, where $h$ may be regarded as the size of a finite element mesh; $I \subseteq(0, \infty)$ with $0 \in \bar{I}$. Note that $H_{h}$ and $L_{h}$ correspond to a triangulation of $\Omega$, while $H_{h}^{-1 / 2}$ corresponds to a triangulation of the interface $\Gamma$. We assume the approximation property, i.e. for any $(u, p, t) \in H$ 
$\times L \times H^{-1 / 2}$, we have

$$
0=\lim _{I \ni h \rightarrow 0} \operatorname{dist}_{H \times L \times H^{-1 / 2}}\left((u, p, t), H_{h} \times L_{h} \times H_{h}^{-1 / 2}\right) .
$$

In addition, we assume for all $h_{1}, h_{2} \in I$ that

$$
h_{1}<h_{2} \Rightarrow H_{h_{2}}^{-1 / 2} \subseteq H_{h_{1}}^{-1 / 2} \text {. }
$$

For $h \in I$ let

$$
i_{h}: H_{h} \subseteq H, \quad j_{h}: H_{h}^{-1 / 2} \hookrightarrow H^{-1 / 2}, \quad \ell_{h}: L_{h} \subseteq L
$$

denote the canonical imbeddings with their duals $i_{h}^{*} \in \mathscr{L}\left(H^{*} ; H_{h}^{*}\right), j_{h}^{*} \in \mathscr{L}\left(H^{1 / 2}\right.$; $\left.\left(H_{h}^{-1 / 2}\right)^{*}\right), \ell_{h}^{*} \in \mathscr{L}\left(L ; L_{h}\right)$.

Remark 4. A natural discretization of problem (P) is obtained taking, for instance, the variational inequality (34) and replacing $H \times L$ by $H_{h} \times L_{h}$. This requires the numerical calculation of $S_{h}:=i_{h}^{*} S i_{h}$ and hence the numerical computation of $V_{2}^{-1}$. Since an explicit representation of $V_{2}^{-1}$ is not known, we are led to approximate $V_{2}^{-1}$ as well introducing a discrete subspace $H_{h}^{-1 / 2}$ for the tractions on the interface.

In order to approximate $S_{h}$, we define

$$
\tilde{S}_{h}:=i_{h}^{*} \gamma^{*} D_{2} \gamma i_{h}+i_{h}^{*} \gamma^{*}\left(\frac{1}{2}-\Lambda^{\prime}\right) j_{h}\left(j_{h}^{*} V_{2} j_{h}\right)^{-1} j_{h}^{*}\left(\frac{1}{2}-\Lambda\right) \gamma i_{h} .
$$

The computation of $\tilde{S}_{h}$ requires the numerical solution of a linear system with a symmetric, positive-definite matrix $V_{2 h}:=\left(j_{h}^{*} V_{2} j_{h}\right)$. In general, $\tilde{S}_{h} \neq i_{h}^{*} S i_{h}$, which causes additional difficulties in the convergence analysis of the numerical treatment of the interface problem [10].

Define the bilinear form

$$
a_{h}:\left\{\begin{array}{l}
\left(H_{h} \times L_{h}\right) \times\left(H_{h} \times L_{h}\right) \rightarrow \mathbb{R}, \\
\left(\left(\begin{array}{l}
u_{h} \\
p_{h}
\end{array}\right),\left(\begin{array}{l}
v_{h} \\
q_{h}
\end{array}\right)\right) \mapsto\left\langle A_{h}\left(\begin{array}{l}
u_{h} \\
p_{h}
\end{array}\right),\left(\begin{array}{l}
v_{h} \\
q_{h}
\end{array}\right)\right\rangle_{H_{h}} \times L_{h}
\end{array},\right.
$$

by

$$
\mathscr{A}_{h}:=\left(\begin{array}{cc}
\tilde{S}_{h}+\varepsilon^{*} A \varepsilon & -\varepsilon^{*} A \\
-A \varepsilon & A+\eta I
\end{array}\right) .
$$

Let $p_{0 h}:=\Pi_{h} p_{0}, \Pi_{h}$ being the orthogonal projection in $L$ onto $L_{h}$.

Definition 3. Given $h \in I$ problem $\left(P_{h}\right)$ consists in finding $\left(u_{h}, p_{h}\right) \in H_{h} \times L_{h}$ with

$$
\begin{aligned}
& a_{h}\left(\left(\begin{array}{c}
u_{h} \\
p_{h}-p_{0 h}
\end{array}\right),\left(\begin{array}{c}
v_{h}-u_{h} \\
q_{h}-p_{h}+p_{0 h}
\end{array}\right)\right)+j\left(q_{h}\right)-j\left(p_{h}-p_{0 h}\right) \\
& -l\left(\left(\begin{array}{c}
v_{h}-u_{h} \\
q_{h}-p_{h}+p_{0 h}
\end{array}\right)\right) \geqslant 0
\end{aligned}
$$

for all $\left(\begin{array}{c}v_{h} \\ q h\end{array}\right) \in H_{h} \times L_{h}$.

Remark 5. If we introduce a new variable $\phi_{h} \in H_{h}^{-1 / 2}$, with $\phi_{h}=V_{2 h}^{-1} j_{h}^{*}\left(\frac{1}{2}-\Lambda_{2}\right) u_{h}$, then (39) would become

$$
\begin{aligned}
0 \leqslant & b_{h}\left(\left(u_{h}, \phi_{h}, p_{h}-p_{0 h}\right),\left(v_{h}-u_{h}, \psi_{h}-\phi_{h}, q_{h}-p_{h}+p_{0 h}\right)\right) \\
& +j\left(q_{h}\right)-j\left(p_{h}-p_{0 h}\right)-l\left(v_{h}-u_{h}, q_{h}-p_{h}+p_{0 h}\right)
\end{aligned}
$$


for the discrete solution $\left(u_{h}, \phi_{h}, p_{h}\right) \in H_{h} \times H_{h}^{-1 / 2} \times L_{h}$ and for all $\left(v_{h}, \psi_{h}, q_{h}\right) \in H_{h}$ $\times H_{h}^{-1 / 2} \times L_{h}$. Here the bilinear form $b_{h}:\left(H_{h} \times H_{h}^{-1 / 2} \times L_{h}\right) \times\left(H_{h} \times H_{h}^{-1 / 2} \times L_{h}\right) \rightarrow \mathbb{R}$ is defined by

$$
b_{h}\left(\left(u_{h}, \phi_{h}, p_{h}\right),\left(v_{h}, \psi_{h}, q_{h}\right)\right):=\left\langle\mathscr{B}\left(\begin{array}{c}
u_{h} \\
\phi_{h} \\
p_{h}
\end{array}\right),\left(\begin{array}{c}
v_{h} \\
\psi_{h} \\
q_{h}
\end{array}\right)\right\rangle_{H_{h} \times H_{h}^{-1 / 2} \times L_{h}},
$$

$\left(u_{h}, \phi_{h}, p_{h}\right),\left(v_{h}, \psi_{h}, q_{h}\right) \in H_{h} \times H_{h}^{-1 / 2} \times L_{h}$ and

$$
\mathscr{B}=\left(\begin{array}{ccc}
\varepsilon^{*} A \varepsilon+\gamma^{*} D_{2} \gamma & \gamma^{*}\left(1 / 2-\Lambda_{2}^{\prime}\right) & -\varepsilon^{*} A \\
\left(1 / 2-\Lambda_{2}\right) \gamma & -V_{2} & 0 \\
-A \varepsilon & 0 & A+\eta I
\end{array}\right) .
$$

The first crucial point in the convergence analysis is the following result.

Lemma 8. There exist $c_{0}>0$ and $h_{0} \in I$ such that for any $h \in I$ with $h<h_{0}$ and any $u_{h} \in H_{h}$ there holds

$$
\left\langle\tilde{S}_{h} u_{h}, u_{h}\right\rangle_{H} \geqslant c_{0}\left\|\gamma u_{h}\right\|_{H^{3,2}}^{2} .
$$

Proof. Assume that (41) is false. Then there exists some parameter sequence $\left(h_{n}\right)$ in $I$ with $\lim _{n \rightarrow \infty} h_{n}=0$ such that for any $n$ there exists $u_{n} \in H_{h_{n}}$ with $\left\|\gamma u_{n}\right\|_{H^{1 / 2}}=1$ and

$$
\left\langle\tilde{S_{h_{n}}} u_{n}, u_{n}\right\rangle \leqslant \frac{1}{n} \text {. }
$$

As shown in [10, Lemma 4.5], $D_{2}$ is positive-semi-definite and a compact perturbation of a positive-definite operator. Thus, the kernel $X:=\operatorname{ker} D_{2}$ has finite dimension and hence is complemented in $H^{1 / 2}$, i.e. there exists a subspace $Y$ of $H^{1 / 2}$ with $X \oplus Y=H^{1 / 2}$. Let $\gamma u_{n}=v_{n}+w_{n}$ with $v_{n} \in X$ and $w_{n} \in Y$. Since $D_{2}$ is positive-definite on $Y$ and $\left\langle\tilde{S}_{h_{n}} u_{n}, u_{n}\right\rangle \geqslant\left\langle D_{2} \gamma u_{n}, \gamma u_{n}\right\rangle$, one concludes from (42) that $\lim _{n \rightarrow \infty} w_{n}=0$. Since $X$ is finite-dimensional, there exists a subsequence (again denoted by $\left(v_{n}\right)$ ) which converges towards some $v \in X$. Thus,

$$
\lim _{n \rightarrow \infty} \gamma u_{n}=v \in X, \quad \text { whence }\|v\|_{H^{1: 2}}=1 \text {. }
$$

Define $x_{n}:=\left(\frac{1}{2}-\Lambda_{2}\right) \gamma u_{n} \in H^{1 / 2}, y_{n}:=j_{h_{n}}^{*} x_{n} \in\left(H_{h_{n}}^{-1 / 2}\right)^{*}$ and $z_{n}:=V_{2 h_{n}}^{-1} y_{n} \in H_{h_{n}}^{-1 / 2}$. Since $\Lambda_{2}$ is continuous, we get

$$
\lim _{n \rightarrow \infty} x_{n}=x:=\left(\frac{1}{2}-\Lambda_{2}\right) v \text {. }
$$

On the other hand, according to the definition of $\tilde{S}_{h},(42)$ implies that

$$
0=\lim _{n \rightarrow \infty}\left\langle y_{n}, V_{2 h}^{-1} y_{n}\right\rangle=\lim _{n \rightarrow \infty}\left\langle V_{2} z_{n}, z_{n}\right\rangle .
$$

Since $V_{2}$ is positive-definite this yields $\lim _{n \rightarrow \infty} z_{n}=0$. Because $V_{2 h}$ is bounded (independent of $h$ ),

$$
\lim _{n \rightarrow \infty} y_{n}=\lim _{n \rightarrow \infty} V_{2 h_{n}} z_{n}=0
$$


Define $Y_{n}:=j_{h_{n}}^{*} x \in\left(H_{h_{n}}^{-1 / 2}\right)^{*}$. Then, by $\lim _{n \rightarrow \infty} x_{n}=x$,

$$
0=\lim _{n \rightarrow \infty}\left\|Y_{n}-y_{n}\right\|_{\left(H_{n_{n}}^{-1 / 2}\right)^{*}}
$$

Let $\phi \in \bigcup_{h \in I} H_{h}^{-1 / 2}$. Then, because of (38), there exists some $n_{0}$ such that $\phi \in H_{h_{n}}^{-1 / 2}$ for any $n \geqslant n_{0}$. Therefore, (45) and (44) lead to

$$
0=\lim _{n \rightarrow \infty}\left\langle\phi, Y_{n}-y_{n}\right\rangle=\langle\phi, x\rangle .
$$

Since $\bigcup_{h \in I} H_{h}^{-1 / 2}$ lies dense in $\left(H^{1 / 2}\right)^{*}=H^{-1 / 2}$, this shows $x=\left(\frac{1}{2}-\Lambda_{2}\right) v=0$.

Thus, according to the definition of $S_{2}$, we get $S_{2} v=0$. Since $S_{2}$ is positive-definite (cf. Lemma 4), $v=0$, which contradicts (43).

The second crucial point in the convergence analysis is the following estimate concerning $\tilde{S_{h}}-S$.

Lemma 9. There exists a constant $c>0$ such that for all $h \in I$ and for all $v_{h}, u_{h} \in H_{h}$ and $u \in H$ there holds

$$
\begin{aligned}
& \left\langle\tilde{S}_{h} v_{h}-i_{h}^{*} S u, v_{h}-u_{h}\right\rangle_{H_{h}} \leqslant c \cdot\left\|v_{h}-u_{h}\right\|_{H} \\
& \quad \cdot\left(\operatorname{dist}_{H^{-1 / 2}}\left(V_{2}^{-1}\left(1 / 2-\Lambda_{2}\right) \gamma u, H_{h}^{-1 / 2}\right)+\left\|v_{h}-u\right\|_{H}\right) .
\end{aligned}
$$

Proof. Firstly, following the notion of [10], we prove

$$
\left\|\left(S_{h}-\tilde{S}_{h}\right) v_{h}\right\|_{H_{h}^{*}} \leqslant c_{1} \cdot \operatorname{dist}_{H^{-1 / 2}}\left(V_{2}^{-1}\left(1 / 2-\Lambda_{2}\right) \gamma v_{h}, H_{h}^{-1 / 2}\right)
$$

for a constant $c_{1}$ which is independent of $h$. Setting $f:=\left(1 / 2-\Lambda_{2}\right) \gamma v_{h}$ and $f_{h}:=j_{h}^{*} f$, one obtains

$$
\left\|\left(S_{h}-\tilde{S}_{h}\right) v_{h}\right\| \leqslant\left\|\left(1 / 2-\Lambda_{2}\right)\right\| \cdot\left\|V_{2}^{-1} f-V_{2 h}^{-1} f_{h}\right\|_{H^{-1}} .
$$

Note that $y:=V_{2}^{-1} f$ is the exact solution of the equation $V_{2} y=f$, while $y_{h}:=V_{2 h}^{-1} f_{h}$ is the solution of the related Galerkin equations $V_{2 h} y_{h}=j_{h}^{*} f$. Since $V_{2}$ is positivedefinite, Céa's lemma yields the quasi-optimal error estimate

$$
\left\|y-y_{h}\right\| \leqslant c_{2} \cdot \operatorname{dist}_{H^{1 / 2}}\left(y, H_{h}^{-1 / 2}\right)
$$

which proves (47).

Using (47) and boundedness of $S$, one gets

$$
\begin{aligned}
& \left\langle\tilde{S}_{h} v_{h}-i_{h}^{*} S u, v_{h}-u_{h}\right\rangle_{H_{h}} \\
& \leqslant\left\|\tilde{S}_{h} v_{h}-i_{h}^{*} S u\right\|_{H_{h}^{*}}\left\|v_{h}-u_{h}\right\|_{H} \\
& \leqslant\left\|v_{h}-u_{h}\right\|_{H} \cdot\left(\left\|\tilde{S}_{h} v_{h}-i_{h}^{*} S v_{h}\right\|_{H_{h}^{*}}+\left\|i_{h}^{*} S\left(u-v_{h}\right)\right\|_{H_{h}^{*}}\right) \\
& \leqslant c_{3}\left\|v_{h}-u_{h}\right\|_{H} \cdot\left(\operatorname{dist}_{H^{-1 / 2}}\left(V_{2}^{-1}\left(1 / 2-\Lambda_{2}\right) \gamma v_{h}, H_{h}^{-1 / 2}\right)+\left\|u-v_{h}\right\|_{H}\right) \\
& \leqslant c_{4}\left\|v_{h}-u_{h}\right\|_{H} \cdot\left(\operatorname{dist}_{H^{-1 / 2}}\left(V_{2}^{-1}\left(1 / 2-\Lambda_{2}\right) \gamma u, H_{h}^{-1 / 2}\right)+\left\|u-v_{h}\right\|_{H}\right) \text {, }
\end{aligned}
$$

where the constants $c_{3}, c_{4}$ are independent of $h$ and we use $\operatorname{dist}\left(f, H_{h}^{-1 / 2}\right) \leqslant \operatorname{dist}\left(g, H_{h}^{-1 / 2}\right)+\|f-g\|_{H^{-1 / 2}}\left(f, g \in H^{-1 / 2}\right)$. This proves the lemma.

Because of the approximation property of the discrete spaces, the next error estimate proves quasi-optimal convergence of the Galerkin procedure. 
Theorem 4. There exist some $c_{0}>0$ and $h_{0} \in I$ such that for any $h \in I$ with $h<h_{0}$ problem $\left(\mathrm{P}_{h}\right)$ has a unique solution $\left(u_{h}, p_{h}\right) . I f(u, p) \in H \times L$ is the solution of problem $(\mathrm{P})$ then there holds

$$
\begin{aligned}
& \left\|\left(\begin{array}{c}
u-u_{h} \\
p-p_{h}-p_{0}+p_{0 h}
\end{array}\right)\right\|_{H \times L}^{2} \leqslant c_{0} \cdot\left\{\operatorname{dist}\left(u, H_{h}\right)^{2}+\operatorname{dist}\left(V_{2}^{-1}\left(1 / 2-\Lambda_{2}\right) \gamma u, H_{h}^{-1 / 2}\right)^{2}\right. \\
& \left.\quad+\inf _{q_{h} \in L_{h}}\left(\left\|p-p_{0}-q_{h}\right\|_{L}^{2}+\left\|p-p_{0}-q_{h}\right\|_{L^{1}\left(\Omega, \mathbb{R}_{s, \mathrm{~m}}^{3 \times 3}\right)}\right)\right\}
\end{aligned}
$$

Proof. Using Lemmas 8 and 6 for the discrete spaces $H_{h} \times L_{h} \times H_{h}^{-1 / 2}$ for $h \in I, h<h_{0}$, one concludes that $\mathscr{A}_{h}$ is positive-definite. Moreover, the related constant $c_{1}>0$ is independent of $h<h_{0}$, i.e. there exists $c_{1}>0$ such that for any $h \in I$ with $h<h_{0}$ and for any $\left(v_{h}, q_{h}\right) \in H_{h} \times L_{h}$ there holds

$$
\left\langle\mathscr{A}_{h}\left(\begin{array}{l}
v_{h} \\
q_{h}
\end{array}\right),\left(\begin{array}{c}
v_{h} \\
q_{h}
\end{array}\right)\right\rangle_{H \times L} \geqslant c_{1}\left\|\left(\begin{array}{c}
v_{h} \\
q_{h}
\end{array}\right)\right\|_{H \times L}^{2} .
$$

As in Theorem 3 this leads to the existence and uniqueness of a solution $\left(u_{h}, p_{h}\right)$ of the discrete problem $\left(\mathbf{P}_{h}\right)$.

Let $v_{h}:=\Pi_{H_{h}} u, \Pi_{H_{h}}$ being the orthogonal projection in $H$ onto $H_{h}$. Then $\left\|u-v_{h}\right\|_{H}$ is bounded above by the right-hand side of (50). Therefore, and because of (51), for the proof of $(50)$, it suffices to show that

$$
\left\langle\mathscr{A}_{h}\left(\begin{array}{c}
v_{h}-u_{h} \\
p-p_{h}-p_{0}+p_{0 h}
\end{array}\right),\left(\begin{array}{c}
v_{h}-u_{h} \\
p-p_{h}-p_{0}+p_{0 h}
\end{array}\right)\right\rangle_{H \times L}
$$

is bounded above by the right-hand side of (50). This will be done in the sequel.

Addition of (34) with $(v, q)=\left(u_{h}, p_{h}-p_{0 h}\right)$ and (39) where $v_{h}=\Pi_{H_{h}} u$ and $q_{h}$ is still arbitrary gives

$$
\begin{aligned}
0 \leqslant & a\left(\left(\begin{array}{c}
u \\
p-p_{0}
\end{array}\right),\left(\begin{array}{c}
u_{h}-u \\
p_{h}-p_{0 h}-p+p_{0}
\end{array}\right)\right)+a_{h}\left(\left(\begin{array}{c}
u_{h} \\
p_{h}-p_{0 h}
\end{array}\right),\left(\begin{array}{c}
v_{h}-u_{h} \\
q_{h}-p_{h}+p_{0 h}
\end{array}\right)\right) \\
& +l\left(\left(\begin{array}{c}
u-v_{h} \\
p-p_{0}-q_{h}
\end{array}\right)\right)+j\left(q_{h}\right)-j\left(p-p_{0}\right) \\
= & -a\left(\left(\begin{array}{c}
u \\
p-p_{0}
\end{array}\right),\left(\begin{array}{c}
u-v_{h} \\
p-p_{0}-q_{h}
\end{array}\right)\right)+l\left(\left(\begin{array}{c}
u-v_{h} \\
p-p_{0}-q_{h}
\end{array}\right)\right)+j\left(q_{h}-p+p_{0}\right) \\
& +a_{h}\left(\left(\begin{array}{c}
u_{h} \\
p_{h}-p_{0 h}
\end{array}\right),\left(\begin{array}{c}
v_{h}-u_{h} \\
q_{h}-p_{h}+p_{0 h}
\end{array}\right)\right)-a\left(\left(\begin{array}{c}
u \\
p-p_{0}
\end{array}\right),\left(\begin{array}{c}
v_{h}-u_{h} \\
q_{h}-p_{h}+p_{0 h}
\end{array}\right)\right) \\
& +j\left(q_{h}\right)-j\left(p-p_{0}\right)-j\left(q_{h}-p+p_{0}\right) .
\end{aligned}
$$

Using the triangle inequality $j\left(p-p_{0}-q_{h}\right)=j\left(q_{h}-p+p_{0}\right) \geqslant j\left(q_{h}\right)-j\left(p-p_{0}\right)$, one obtains that (55) is less than or equal to zero. According to Lemma $7,(53)$ is equal to

$$
j\left(q_{h}-p+p_{0}\right)+\int_{\Omega} k \lambda\left(p-p_{0}-q_{h}\right) \mathrm{d} \Omega
$$


which is less than or equal to

$$
2 j\left(q_{h}-p+p_{0}\right) \leqslant 2 k_{2} \cdot\left\|p-p_{0}-q_{h}\right\|_{L^{1}\left(\Omega ; R_{\mathrm{sym}}^{3 \times 3}\right)} \cdot
$$

In view of the above inequality concerned with (53)-(55), we may add these terms to (52) and obtain a new upper bound of (52) which can be rewritten as

$$
\begin{aligned}
2 k_{2} & \left\|p-p_{0}-q_{h}\right\|_{L^{1}\left(\Omega ; \mathbb{R}_{s y m}^{3 \times 3}\right)}+\left\langle\tilde{S}_{h} v_{h}-S u, v_{h}-u_{h}\right\rangle_{H_{h}^{-1 / 2}} \\
& +\left\langle\varepsilon^{*} A \varepsilon\left(v_{h}-u\right), v_{h}-u_{h}\right\rangle_{H}+\left\langle A \varepsilon\left(u-v_{h}\right), p-p_{0}-p_{h}+p_{0 h}\right\rangle_{L} \\
& +\left\langle A \varepsilon\left(u-u_{h}\right), q_{h}-p+p_{0}\right\rangle_{L} \\
& +\left\langle(A+\eta I)\left(p-p_{0}-q_{h}\right), p-p_{0}-p_{h}+p_{0 h}\right\rangle_{L} .
\end{aligned}
$$

Using Lemma 9, the boundedness of some operators and (51), the above estimate of (52) leads to

$$
\begin{aligned}
& \frac{1}{c}\left\|\left(\begin{array}{c}
v_{h}-u_{h} \\
p-p_{h}-p_{0}+p_{0 h}
\end{array}\right)\right\|_{H \times L}^{2} \\
& \quad \leqslant \quad\left\|p-p_{0}-q_{h}\right\|_{L^{1}\left(\Omega ; \mathbb{R}_{\mathrm{vym}}^{3 \times 3}\right)} \\
& \quad+\left\|v_{h}-u_{h}\right\|_{H} \cdot\left(\operatorname{dist}_{H^{-1 / 2}}\left(V_{2}^{-1}\left(1 / 2-\Lambda_{2}\right) \gamma u, H_{h}^{-1 / 2}\right)+\left\|v_{h}-u\right\|_{H}\right) \\
& \quad+\left\|\left(\begin{array}{c}
v_{h}-u \\
p-p_{0}-q_{h}
\end{array}\right)\right\|_{H \times L}\left\|\left(\begin{array}{c}
u-u_{h} \\
p-p_{0}-p_{h}+p_{O h}
\end{array}\right)\right\|_{H \times L} \\
& \leqslant \\
& \quad+p-p_{0}-q_{h}\left\|_{L^{1}\left(\Omega ; \mathbb{R}_{\mathrm{yym}}^{3 \times 3}\right)}+c\right\| p-p_{0}-q_{h} \|_{H \times L}^{2} \\
& \quad+\left(2 c+\frac{1}{2 c}\right)\left\|\left(\begin{array}{c}
v_{h}-u_{h} \\
p-p_{0}-p_{h}+p_{0 h}
\end{array}\right)\right\|_{H \times L}^{2} \\
& \quad+2 c\left(\operatorname{dist}_{H^{-1 / 2}}\left(V_{2}^{-1}\left(1 / 2-\Lambda_{2}\right) \gamma u, H_{h}^{-1 / 2}\right)+\left\|v_{h}-u\right\|_{H}\right)^{2},
\end{aligned}
$$

where we used $\left\|v_{h}-u_{h}\right\|_{H}^{2} \leqslant\left\|u-u_{h}\right\|_{H}^{2}=\left\|u-u_{h}\right\|_{H}^{2}+\left\|u_{h}-v_{h}\right\|_{H}^{2}$. From this one concludes (50).

Remark 6. Theorem 4 extends the error estimate [16, Equation (3.4)] for the interior problem to the non-linear interface problem. There, the estimate (50) is applied to particular trial spaces assuming a certain regularity of the solutions. These considerations apply also in the present case.

Remark 7. The discrete problem $\left(\mathrm{P}_{h}\right)$ must be solved by an iterative procedure like e.g. Uzawa's algorithm. For a discussion of regularization procedures one is referred to $[16,20]$.

Remark 8. Using the extended discrete problem (40), one can also consider the error of the tractions. From the proof of Lemma 9 it can be observed that $\left\|\phi-\phi_{h}\right\|_{H_{h}^{-1 / 2}}^{2}$ is also bounded by the right-hand side of (50).

\section{References}

1. Bielak, J. and MacCamy, R. C., 'An exterior interface problem in two-dimensional elastodynamics', Quart. Appl. Math. 41, 143-159 (1983).

2. Brezzi, F. and Johnson, C., 'On the coupling of boundary integral and finite element methods', Calcolo 16, 189-201 (1979). 
3. Ciarlet, P. G., The Finite Element Method for Elliptic Problems, North-Holland, Amsterdam, 1978.

4. Costabel, M., 'Symmetric methods for the coupling of finite elements and boundary elements', in: Boundary Elements IX, Eds. C. A. Brebia et al., Vol. 1, Springer, Berlin, 1987, pp. 411-420.

5. Costabel, M., 'Boundary integral operators on Lipschitz domains: elementary results, SIAM J. Math. Anal. 19, 613-626 (1988).

6. Costabel, M., 1992, private communication.

7. Costabel, M. and Stephan, E. P., 'Boundary integral equations for mixed boundary value problems in polygonal domains and Galerkin approximation', Banach Center Publ. 15, 175-251 (1985).

8. Costabel, M. and Stephan, E. P., 'A direct boundary integral equation method for transmission problems', J. Math. Anal. Appl. 106, 367-413 (1985).

9. Costabel, M. and Stephan, E. P., 'Integral equations for transmission problems in linear elasticity', $J$. Integr. Eq. Appl. 2, 211-223 (1990).

10. Costabel, M. and Stephan, E. P., 'Coupling of finite and boundary element methods for an elastoplastic interface problem', SIAM J. Numer. Anal. 27, 1212-1226 (1990).

11. Ekeland, I. and Temam, R., Convex Analysis and Variational Problems, North-Holland, Amsterdam, 1976.

12. Gatica, G. N. and Hsiao, G. C., 'The coupling of boundary element and finite element methods for a nonlinear exterior boundary value problem', Z. Anal. Anw. 8, 377-387 (1989).

13. Gatica, G. N. and Hsiao, G. C. 'On the coupled BEM and FEM for a nonlinear exterior Dirichlet problem in $R^{2}$, Numer. Math. 61, 171-214 (1992).

14. Glowinski, R., Lions, J. L. and Trémolières, R., Numerical Analysis of Variational Inequalities, NorthHolland, Amsterdam, 1981.

15. Han, H., 'A new class of variational formulations for the coupling of finite and boundary element methods', J. Comput. Math. 8, 223-232 (1990).

16. Han, W., 'Finite element analysis of a holonomic elastic-plastic problem', Numer. Math. 60, 493-508 (1992).

17. Hsiao, G. C., Stephan, E. P. and Wendland, W. L., 'On the Dirichlet problem in elasticity for a domain exterior to an arc', J. CAM 34, (1991) 1-19.

18. Johnson, C. and Nedelec, J. C., 'On the coupling of boundary integral and finite element methods', Math. Comp. 35, 1063-1079 (1980).

19. Kupradze, V. D., et al., Three-dimensional Problems of the Mathematical Theory of Elasticity and Thermoelasticity, North-Holland, Amsterdam, 1979.

20. Reddy, B. D. and Griffin, T. B., 'Variational principles and convergence of finite element approximations of a holonomic elastic-plastic problem', Numer. Math. 52, 101-117 (1988).

21. Reddy, B. D., Griffin, T. B. and Marais, M. J., 'A penalty approach to the rate problem in small-strain plasticity', IMA J. Appl. Math. 34, 303-321 (1985).

22. Reddy, B. D., Martin J. B. and Griffin, T. B., 'Extremal paths and holonomic constitutive laws in elastoplasticity', $Q$. Appl. Math. 45, 487-502 (1987).

23. Wendland, W. L., 'On asymptotic error estimates for combined BEM and FEM', in Finite and Boundary Element Techniques From Mathematical and Engineering Point of View, Eds. E. Stein and W. L. Wendland, CISM Courses 301. Springer, New York, 1988, pp. 273-331.

24. Zeidler, E., Nonlinear Functional Analysis and its Applications II, Vols. A and B, Springer, New York, 1990.

25. Zeidler, E., Nonlinear Functional Analysis and its Applications III, Springer, New York, 1985.

26. Zeidler, E., Nonlinear Functional Analysis and its Applications IV, Springer, New York, 1988. 